\title{
ARTICLE
}

Received 13 May 2013 | Accepted 14 Aug 2013 | Published 19 Sep 2013 DOI: 10.1038/ncomms3449

\section{Hour-glass magnetic spectrum in a stripeless insulating transition metal oxide}

\author{
Y. Drees ${ }^{1}$ D. Lamago ${ }^{2}$, A. Piovano ${ }^{3} \&$ A.C. Komarek ${ }^{1}$
}

An hour-glass-shaped magnetic excitation spectrum appears to be a universal characteristic of the high-temperature superconducting cuprates. Fluctuating charge stripes or alternative band structure approaches are able to explain the origin of these spectra. Recently, an hourglass spectrum has been observed in an insulating cobaltate, thus favouring the charge stripe scenario. Here we show that neither charge stripes nor band structure effects are responsible for the hour-glass dispersion in a cobaltate within the checkerboard charge-ordered regime of $\mathrm{La}_{2-x} \mathrm{Sr}_{x} \mathrm{CoO}_{4}$. The search for charge stripe ordering reflections yields no evidence for charge stripes in $\mathrm{La}_{1.6} \mathrm{Sr}_{0.4} \mathrm{CoO}_{4}$, which is supported by our phonon studies. With the observation of an hour-glass-shaped excitation spectrum in this stripeless insulating cobaltate, we provide experimental evidence that the hour-glass spectrum is neither necessarily connected to charge stripes nor to band structure effects, but instead, probably intimately coupled to frustration and arising chiral or non-collinear magnetic correlations.

\footnotetext{
${ }^{1}$ Max-Planck-Institute for Chemical Physics of Solids, Physics of Correlated Matter, Nöthnitzer Street 40, D-01187 Dresden, Germany. ${ }^{2}$ Laboratoire Léon Brillouin, CEA/CNRS, F-91191 Gif-sur Yvette, France. ${ }^{3}$ Institut Laue-Langevin (ILL), 6 Rue Jules Horowitz, F-38043 Grenoble, France. Correspondence and requests for materials should be addressed to A.C.K. (email: Komarek@cpfs.mpg.de).
} 
C harge stripes that have been initially predicted as a combined charge- and spin-density wave phenomenon ${ }^{1-3}$ have been first observed experimentally in $\mathrm{Nd}$-codoped $\mathrm{La}_{2}-{ }_{x} \mathrm{Sr}_{x} \mathrm{CuO}_{4}$ (ref. 4) and in the isostructural nickelates $\mathrm{La}_{2-x} \mathrm{Sr}_{x} \mathrm{NiO}_{4+\delta}$ (LSNO) (ref. 5) with a quasi-two-dimensional layered structure. These charge stripes are characterized by holerich regions running in vertical ${ }^{4}$ or diagonal $^{5}$ direction within the $M \mathrm{O}_{2}$ planes $(M=\mathrm{Cu} / \mathrm{Ni})$, thus separating the remaining chargedepleted regions where the antiferromagnetic (AFM) structure of the undoped parent compound recovers. As charge stripes act as antiphase domain walls, the magnetic structure appears modulated. The role of this charge stripe instability for the high-temperature superconducting (HTSC) cuprates remains a matter of debate and it is puzzling that both phenomena can coexist, although charge stripes tend to suppress superconductivity ${ }^{6}$. Another isostructural system where stripe ordering has been reported is $\mathrm{La}_{2}{ }_{x} \mathrm{Sr}_{x} \mathrm{CoO}_{4}$ (refs 7,8). Surprisingly, also an hour-glass magnetic spectrum has been observed in $\mathrm{La}_{5 / 3} \mathrm{Sr}_{1 / 3} \mathrm{CoO}_{4}$ (ref. 8). This hour-glass spectrum resembles the famous excitations in the cuprates ${ }^{9-18}$, which were found to be a universal feature of these HTSC materials and which has stimulated enormous efforts for explaining these spectra ${ }^{19-22}$. Very recently, theoretical simulations of the magnetic excitation spectra based on a disordered charge stripe model have been also reported for $\mathrm{La}_{5 / 3} \mathrm{Sr}_{1 / 3} \mathrm{CoO}_{4}$ (ref. 23). These layered cobalt oxides are isostructural to the prototypical cuprate materials $\mathrm{La}_{2}-{ }_{x} \mathrm{Sr}_{x} \mathrm{CuO}_{4}$. The quasi-two-dimensional crystal structure arises from a stacking of $\mathrm{CoO}_{2}$ layers that are separated by $(\mathrm{La}, \mathrm{Sr}) \mathrm{O}$ rocksalt layers acting as a charge reservoir. The substitution of trivalent $\mathrm{La}$ by divalent $\mathrm{Sr}$ introduces holes into the $\mathrm{CoO}_{2}$ layers. In analogy with the isostructural nickelates (LSNO), these holes were proposed to segregate into diagonal charge stripes ${ }^{7,8}$. However, whereas the incommensurate magnetic peaks can be clearly observed in $\mathrm{La}_{2-x} \mathrm{Sr}_{x} \mathrm{CoO}_{4}$ (ref. 7), the corresponding unambiguous observation of sharp and well-separated charge stripe ordering (CSO) superstructure reflections is missing. Hence, also a glassy charge-ordered state with different commensurate superlattice fragments could be responsible for the observed broad signals in $\mathrm{Pr}_{2-x} \mathrm{Ca}_{x} \mathrm{CoO}_{4}$ (ref. 24) or even simply disordered magnetic contributions spread around half-integer positions in reciprocal space for the reported features in $\mathrm{La}_{2}{ }_{x} \mathrm{Sr}_{x} \mathrm{CoO}_{4}$ (ref. 7).

Here we have studied the magnetism, magnetic excitations, charge ordering and electron-phonon coupling in a cobaltate compound close to half doping, that is, in $\mathrm{La}_{1.6} \mathrm{Sr}_{0.4} \mathrm{CoO}_{4}$ and were able to observe the appearance of an hour-glass dispersion in this stripeless cobaltate within the checkerboard chargeordered regime of $\mathrm{La}_{2}{ }_{x} \mathrm{Sr}_{x} \mathrm{CoO}_{4}$.

\section{Results}

Spin and charge order. In contrast to other studied cobaltates within the incommensurate magnetic regime, our sample exhibits comparably sharp incommensurate magnetic peaks and, thus, much less disorder than in previously studied compounds (see Fig. 1). Note that it is shown in ref. 23 that the sharpness of the CSO signal should be roughly equal to the sharpness of the incommensurate magnetic peaks in a CSO scenario for $\mathrm{La}_{2-x} \mathrm{Sr}_{x} \mathrm{CoO}_{4}$ (ref. 23). Hence, our $\mathrm{La}_{1.6} \mathrm{Sr}_{0.4} \mathrm{CoO}_{4}$ material seems to be an ideal system to study any possible relationship between incommensurate magnetic peaks and charge stripes in these layered cobaltates. The results of our elastic neutronscattering experiments on $\mathrm{La}_{1.6} \mathrm{Sr}_{0.4} \mathrm{CoO}_{4}$ are shown in Fig. 1 . Finally, we were not able to detect any signal indicative for CSO in $\mathrm{La}_{1.6} \mathrm{Sr}_{0.4} \mathrm{CoO}_{4}$; see Fig. 1c. Instead, only one broad peak at half-integer positions appears that is indicative for a (disordered) checkerboard charge-ordered (CBCO) state persisting up to high temperatures very similar to the optimum half-doped compound $\mathrm{La}_{1.5} \mathrm{Sr}_{0.5} \mathrm{CoO}_{4}$ (dashed line). Thus, we managed to find a $\mathrm{La}_{2}-{ }_{x} \mathrm{Sr}_{x} \mathrm{CoO}_{4}$ sample within the $\mathrm{CBCO}$-ordered regime, which exhibits clearly incommensurate magnetism.

Magnetic excitation spectra. Next, we studied the magnetic excitations of this compound. In Fig. 2a-f, the magnetic excitation spectra of $\mathrm{La}_{1.6} \mathrm{Sr}_{0.4} \mathrm{CoO}_{4}$ are shown and compared with those in $\mathrm{La}_{1.5} \mathrm{Sr}_{0.5} \mathrm{CoO}_{4}$. In contrast to the half-doped reference material (the dashed lines in Fig. 2d,f were derived from spin wave calculations using the McPhase program code ${ }^{25}$ closely following the analysis in ref. 26), all basic features of an hour-glass magnetic spectrum can be observed in $\mathrm{La}_{1.6} \mathrm{Sr}_{0.4} \mathrm{CoO}_{4}$-an inwards-dispersion of low-energy branches towards the planar AFM wavevector, a suppression of the outwards-dispersing branches, a resonance-like merging around $\sim 20 \mathrm{meV}$ with increased intensity and an outwards dispersion of the high-energy branches measured at positions rotated by $45^{\circ}$ compared with the elastic magnetic satellites. Additional constantenergy maps for $\mathrm{La}_{1.6} \mathrm{Sr}_{0.4} \mathrm{CoO}_{4}$ are shown in Fig. $2 \mathrm{~g}-\mathrm{O}$. Remarkably, the high-energy magnetic excitations in $\mathrm{La}_{1.6} \mathrm{Sr}_{0.4} \mathrm{CoO}_{4}$ resemble on the isotropic high-energy excitations recently reported for $\mathrm{La}_{1.96} \mathrm{Sr}_{0.04} \mathrm{CuO}_{4}$ (ref. 27). The constant- $Q$ scan at $(3 / 21 / 20)$ clearly shows the resonance-like increase of intensity at the magnon-merging point; see Fig. 2p. Further, we have studied the low-energy magnetic excitations at a cold triple-axis spectrometer (TAS). In Fig. 2q, the energy scan at an incommensurate magnetic peak position is shown, which indicates the possible presence of a gap in the spin excitation spectrum that amounts to about $\sim 1.8$ $\mathrm{meV}$. The incomplete suppression of inelastic magnetic intensities below this possible gap could be explained by an intrinsic peak width of the magnetic modes arising from short-lived damped magnetic excitations (that are probably induced by the high degree of disorder by the random doping of electrons into the CBCO matrix). The vicinity of our $40 \%$ Sr-doped sample to the half-doped parent compound might favour a similiar origin of the gap as in $\mathrm{La}_{1.5} \mathrm{Sr}_{0.5} \mathrm{CoO}_{4}$, that is, a single ion or small exchange anisotropy within the $a b$ plane $^{26}$. In addition, the existence of a gap in these hour-glass-shaped excitations in $\mathrm{La}_{1.6} \mathrm{Sr}_{0.4} \mathrm{CoO}_{4}$ resembles the observations in the isostructural HTSC cuprates (for example, in $\mathrm{La}_{1.88} \mathrm{Sr}_{0.12} \mathrm{CuO}_{4}$ (ref. 28)) even if their origin might be different.

Electron-phonon coupling. We have also searched for signatures of charge ordering in the $\mathrm{Co}-\mathrm{O}$ bond-stretching phonon dispersion of $\mathrm{La}_{2}-{ }_{x} \mathrm{Sr}_{x} \mathrm{CoO}_{4}$. In Fig. 3a, two polarization patterns of high-frequency $\Sigma_{1}$ phonon modes are shown. In the left figure, it is shown schematically that the presence of diagonal charge stripes (indicated by the dashed lines) would induce a coupling of the $\mathrm{Co}-\mathrm{O}$ bond-stretching phonon mode to stripes with the same propagation vector. The fact that charge stripes in single-layer perovskite oxide materials are able to induce such an electronphonon coupling and even giant electron-phonon anomalies was already demonstrated for high-energy $\mathrm{Cu}-\mathrm{O}$ bond-stretching phonon modes in the isostructural cuprates ${ }^{29}$. On the other hand, for $\mathrm{CBCO}$ one would expect bond-stretching phonon anomalies at the zone boundary as is shown schematically in the right part of Fig. 3a. Here we have measured the topmost $\mathrm{Co}-\mathrm{O}$ bondstretching phonon dispersion of $\mathrm{La}_{1.6} \mathrm{Sr}_{0.4} \mathrm{CoO}_{4}$ and several $\mathrm{La}_{2-}{ }_{x} \mathrm{Sr}_{x} \mathrm{CoO}_{4}$ reference samples $(x=0,1 / 3,0.5)$; see Fig. $3 \mathrm{~b}, \mathrm{c}, \mathrm{e}$. Compared with the undoped $\mathrm{Co}^{2+}$ compound $\mathrm{La}_{2} \mathrm{CoO}_{4}$, the $\mathrm{La}_{2}-{ }_{x} \mathrm{Sr}_{x} \mathrm{CoO}_{4}$ materials within the incommensurate magnetic regime all exhibit a strong pronounced softening of the phonon dispersion towards the zone boundary, that is, at (1/2 1/2 0$)$ and not at propagation vectors corresponding to the expected position for CSO at $(\zeta \zeta 0)$ with $\zeta=2 \cdot \varepsilon \sim x$ (indicated by the black arrows 

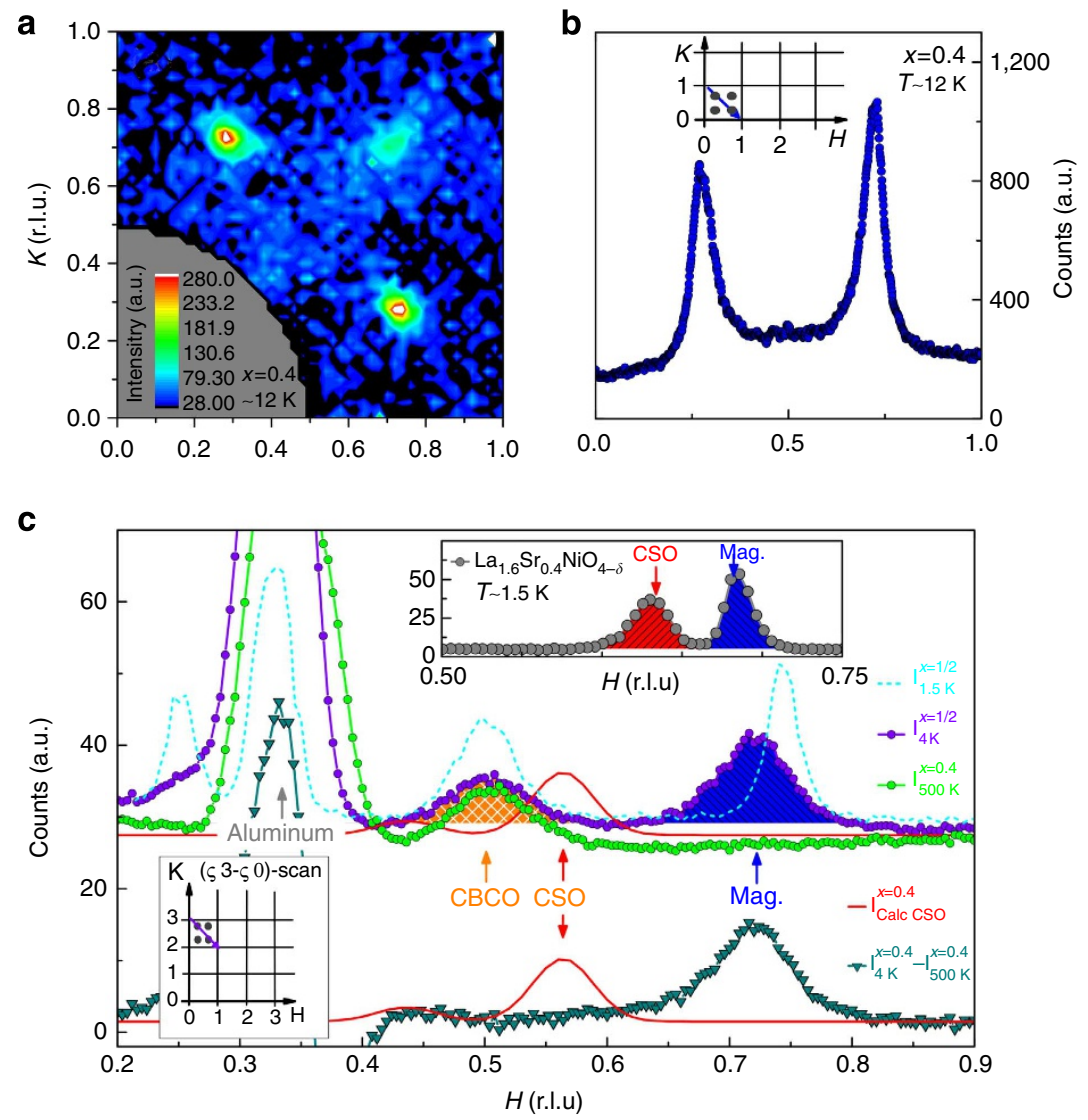

Figure 1 | Elastic neutron diffraction study of charge and magnetic correlations. (a) Neutron-scattering intensities in the $\mathrm{HKO}^{-\mathrm{plane}}$ for $\mathrm{La}_{1.6} \mathrm{Sr}_{0.4} \mathrm{CoO}_{4}$ at $12 \mathrm{~K}$; (200 K background subtracted). (b) Magnetic peaks measured in scans across (1/2 1/2 0). (c) Diagonal ( $\left.\mathrm{H} 3-\mathrm{H} \mathrm{O}_{0}\right) \mathrm{scans}_{\mathrm{s}}$ for $\mathrm{La}_{1.6} \mathrm{Sr}_{0.4} \mathrm{CoO}_{4}$ measured at 4 and at $500 \mathrm{~K}$. In addition, the difference of 4 and $500 \mathrm{~K}$ intensities is shown together with the expected CSO intensity (red line) derived from a CSO-ordered LSNO reference sample (inset) with the same Sr doping $\left(l_{\text {calc CSO }}^{x=0.4}=\left.\alpha\right|_{\text {magn }} ^{x=0.4} \cdot I_{\mathrm{CSO}}^{\mathrm{LSNO}} / I_{\text {magn }}^{\mathrm{LSNO}} ; \alpha=\left(m_{\mathrm{Ni}} / m_{\mathrm{CO}}\right)^{2} \cdot\left(u_{\mathrm{Co}} / u_{\mathrm{Ni}}\right)^{2} \sim 1\right)$. The clear absence of any CSO signal and the broad signal at half-integer positions indicates that our $\mathrm{La}_{1.6} \mathrm{Sr}_{0.4} \mathrm{CoO}_{4}$ sample is still within the $\mathrm{CBCO}$ regime. Dashed cyan line: the charge-ordering signal of the optimum-doped $\mathrm{CBCO}$-ordered sample $\mathrm{La}_{1.5} \mathrm{Sr}_{0.5} \mathrm{CoO}_{4}$. Intensity error bars are statistical error bars calculated by the square root of intensity.

in Fig. 3c,d). In contrast to our observations in $\mathrm{La}_{1.6} \mathrm{Sr}_{0.4} \mathrm{CoO}_{4}$, the corresponding $\mathrm{Ni}-\mathrm{O}$ bond-stretching phonon mode of a $40 \% \mathrm{Sr}-$ doped LSNO reference material with robust diagonal CSO exhibits a completely different and almost complementary dispersion; see Fig. 3d. The high-frequency $\mathrm{Ni}-\mathrm{O}$ bond-stretching mode starts at very similar energies for $\zeta=0$ but quickly softens around propagation vectors corresponding to CSO propagation vectors and even exhibits a final upturn towards the zone boundary. It is noteworthy that we also observed phonon softening in other LSNO samples (with $x=0.2$ ), where the phonon softening appears at phonon propagation vectors that exactly correspond to the propagation vectors of CSO of that particular LSNO sample ${ }^{30}$. Hence, an opposing behaviour of bond-stretching phonon modes in cobaltates and in charge stripe-ordered nickelates can be observed. In the cobaltates, the anomalous phonon softening at the zone boundary is consistent with a robust $\mathrm{CBCO}$ persisting also below half doping rather than with static (or dynamic) charge stripe phases and supports our elastic studies.

\section{Discussion}

The absence of stripes in $\mathrm{La}_{1.6} \mathrm{Sr}_{0.4} \mathrm{CoO}_{4}$ and our observation of an hour-glass-shaped excitation spectrum in this compound (even with indications for a gap) shed another light on the recent argumentation that the absence of any gap in the hour-glass-shaped excitations of the cobaltates $\left(\mathrm{La}_{5 / 3} \mathrm{Sr}_{1 / 3} \mathrm{CoO}_{4}\right)$ with static stripe order and the appearance of a gap in the excitations of the HTSC cuprates point to a 'collective quantum melting of stripe-like electronic order' in the cuprates ${ }^{31}$. Moreover, the study of larger parts of the phase diagram of $\mathrm{La}_{2}{ }_{x} \mathrm{Sr}_{x} \mathrm{CoO}_{4}$ motivates a different mechanism for the emergence of incommensurate magnetism in $\mathrm{La}_{2-x} \mathrm{Sr}_{x} \mathrm{CoO}_{4}(x \sim 1 / 2)$; see Fig. 4 . Apparently, the optimum-doped CBCO-ordered compound $\mathrm{La}_{1.5} \mathrm{Sr}_{0.5} \mathrm{CoO}_{4}$ exhibits the sharpest magnetic peaks, which broaden with increasing distance of hole doping away from half doping. Similar observations have been reported in ref. 7 . With decreasing $\mathrm{Sr}$ doping, the incommensurate magnetic regime finally breaks down slightly below 1/3 doping. Compared with a CSO scenario, the continuously increasing peak width with decreasing $\mathrm{Sr}$ concentration below half doping in the cobaltates is more indicative for a rising amount of frustration in an alternative chiral or non-collinear magnetic scenario: starting from the optimum half-doped composition with sharpest magnetic peaks, the doping of additional electrons ( $\mathrm{Co}^{2}+$ ions) into the ideal CBCO-ordered AFM matrix induces frustration. Thus, the substitution of $\mathrm{Co}^{2+}$ ions for non-magnetic $\mathrm{Co}^{3+}$ ions alters the AFM Co ${ }^{2+\uparrow}-\mathrm{Co}^{3+}-\mathrm{Co}^{2+\downarrow}-\mathrm{Co}^{3+}-\mathrm{Co}^{2+\uparrow}$ exchange paths resulting in frustrated spin arrangements $\mathrm{Co}^{2+\uparrow}-\mathrm{Co}^{2+\downarrow}-\mathrm{Co}^{2+?}-\mathrm{Co}^{3+}-\mathrm{Co}^{2 \uparrow}$; see Fig. $4 \mathrm{~d}$. To relieve 

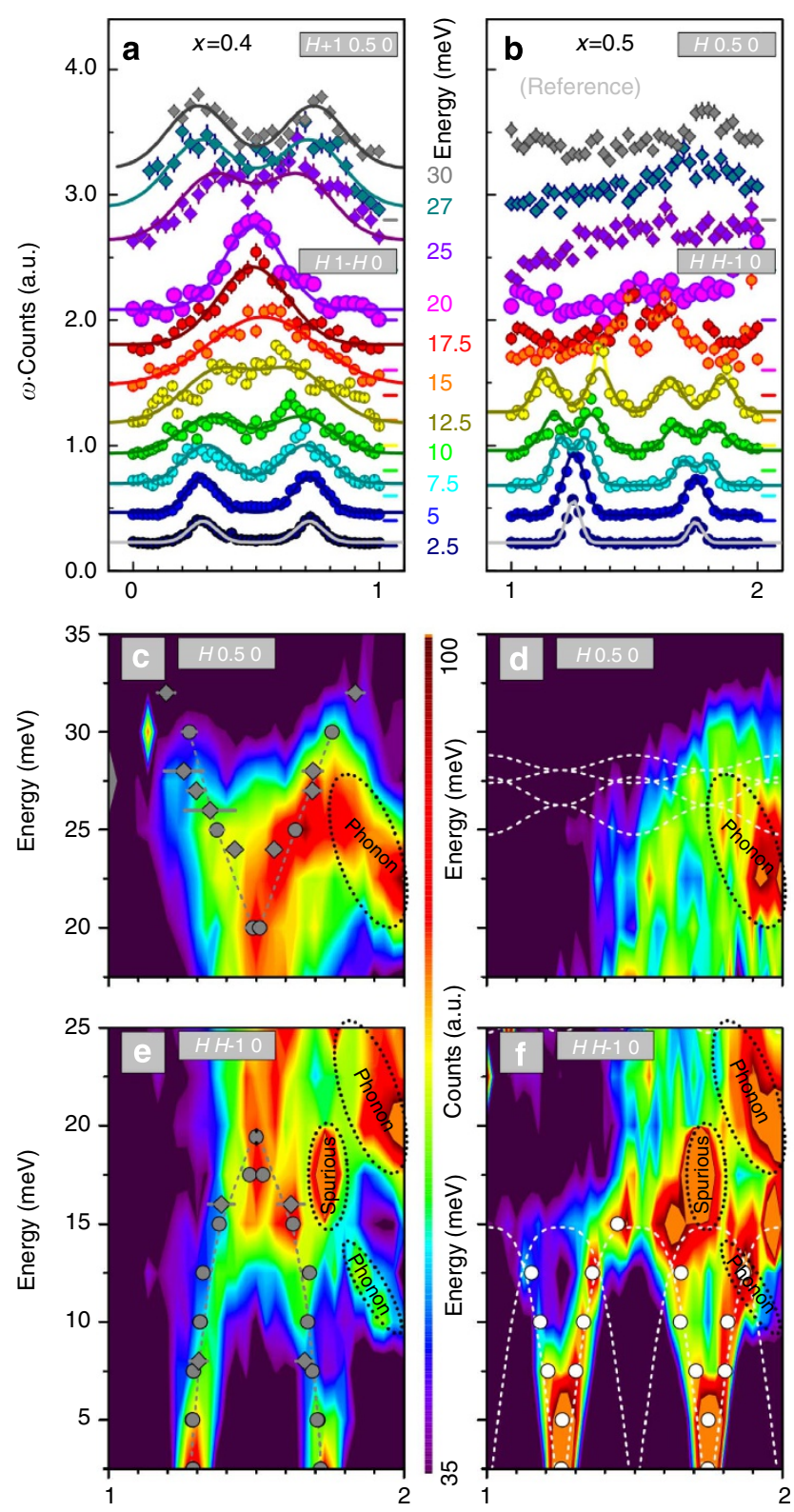

$H$ (r.l.u.)
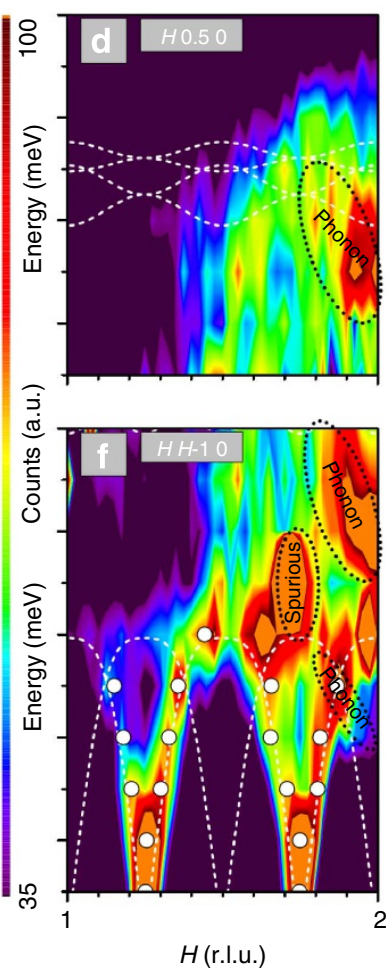

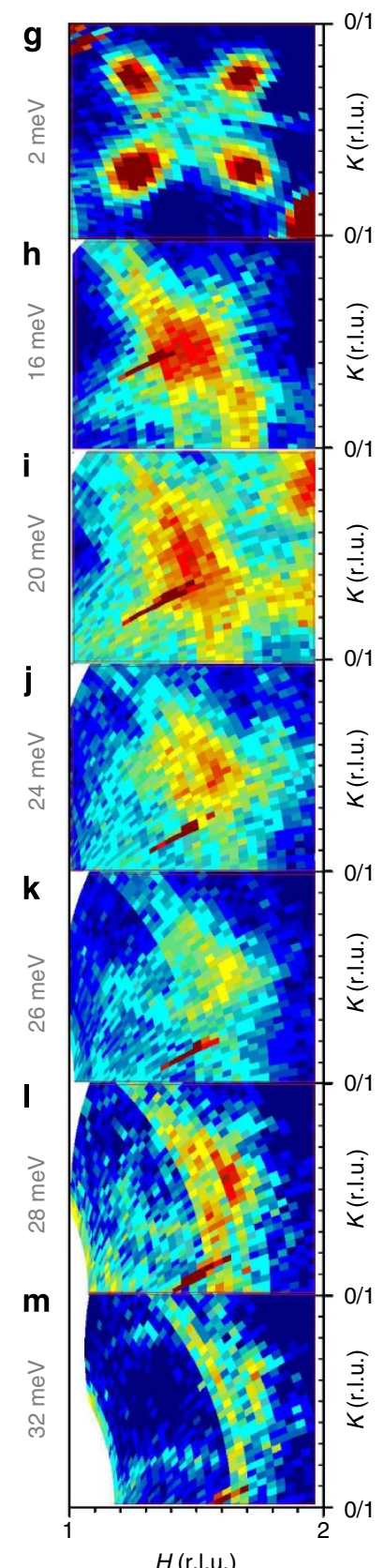

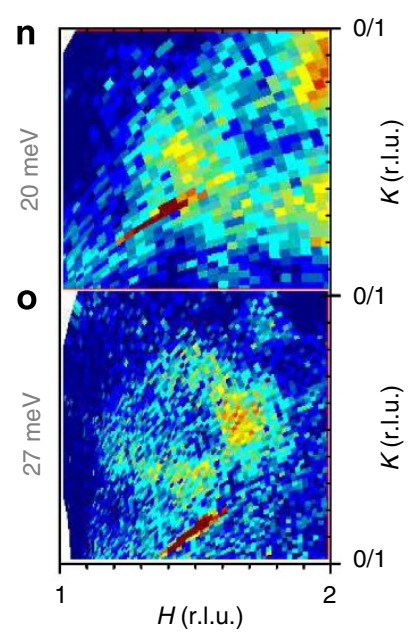

$\mathbf{p}$

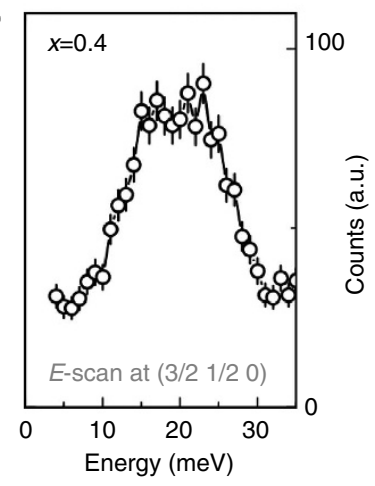

$\mathbf{q}$

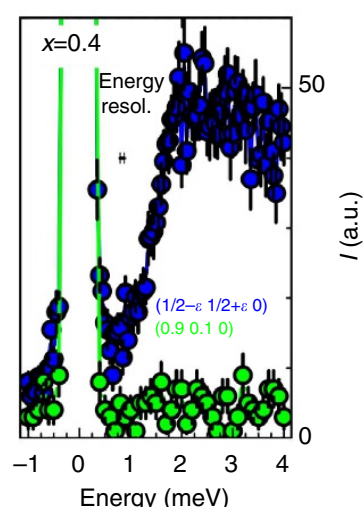

Figure 2 | Neutron spectroscopy study of magnetic excitations. (a-f) Inelastic neutron-scattering intensities for $\mathrm{La}_{1.6} \mathrm{Sr}_{0.4} \mathrm{CoO}_{4}$ and $\mathrm{La}_{1.5} \mathrm{Sr}_{0.5} \mathrm{CoO}_{4}$ measured at the $2 \mathrm{~T}$ spectrometer. (a,b) Neutron-scattering intensities (shifted by a value indicated by the horizontal bars); circles: diagonal scans across $(1 / 21 / 20)$ in $\mathbf{a}$ and $(3 / 21 / 20)$ in $\mathbf{b}$ as a function of $H$; diamonds: ( $H 0.50)$ scans plotted as a function of $H-1$ and $H$ in $\mathbf{a}$ and $\mathbf{b}$, respectively; solid lines were derived from symmetric gaussian fits. (c-f) Intensity maps as a function of $Q$ and energy transfer through (3/21/20) in [10 0] direction (c,d) and [110] direction (e,f). Maps have been generated from the data above (colours between two data points are interpolated). Dashed lines are guide to the eyes (c,e) and dispersions derived from spin wave calculations (d,f); black/dashed circles: spurious peaks/phonons. Error bars for all data included (might be smaller than symbol size). (g-o) Constant-energy maps measured the IN8 spectrometer (energy written to the left of each map). $H(K)$ is ranging from 1 to 2 (from 0 to 1) from left to right (from bottom to top) in each map. Instead of interpolation, the whole area around one measured data point is filled with the same colour. Fits of the dispersion (diamonds) that have been derived from these maps are also included in $\mathbf{c}$ and $\mathbf{e}$. In $\mathbf{n}$ and $\mathbf{o}$, the background was subtracted (see Methods Section). (p) Constant- $Q$ scan at (3/2 1/2 0). (q) Constant- $Q$ scans at $(1 / 2-\varepsilon 1 / 2+\varepsilon 0)$ measured at $\sim 10 \mathrm{~K}$ at a cold neutron TAS (blue circles); in addition, the background is shown (green circles). Intensity error bars are statistical error bars calculated by the square root of intensity. Other error bars are s.d. obtained from Gaussian peak fits.

the high amount of frustration that is generated by the introduction of additional large nearest neighbour exchange couplings $J>>J^{\prime}$, the system can be expected to turn into a chiral or non-collinear magnetic state. This scenario is not only in agreement with our observation of incommensurate magnetism together with the absence of charge stripes but it is also able to explain naturally the successively increasing peak broadening away from half doping; see Fig. 4b. In addition, the kind of incommensurate magnetism naturally follows from our frustration scenario. As shown in Fig. 4d, each doped electron into the undistorted AFM matrix of the ideal 
a

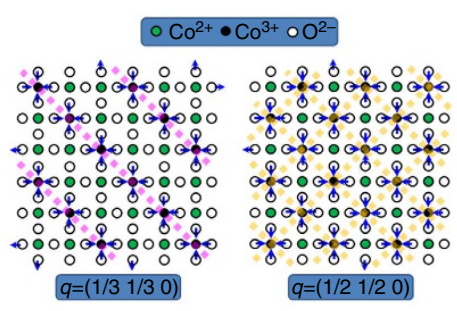

b

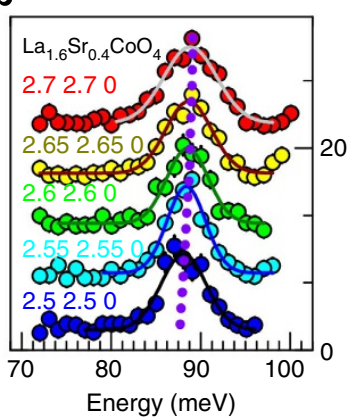

c

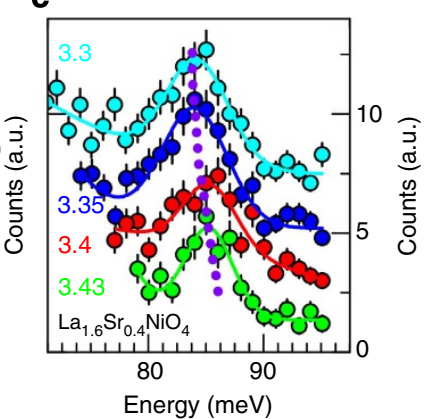

d

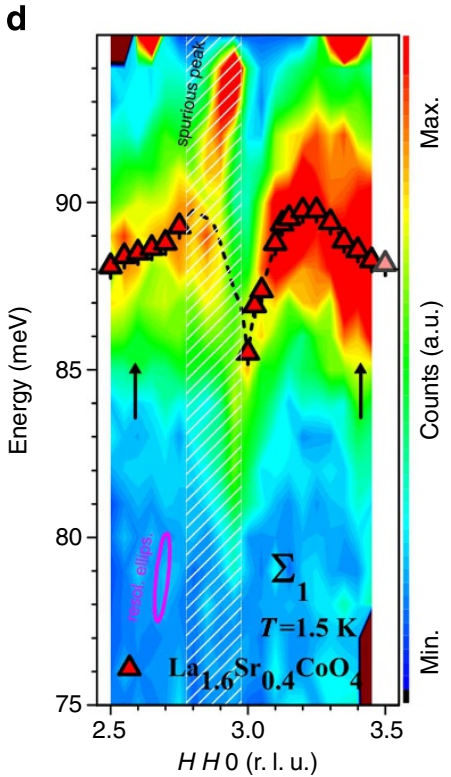

e

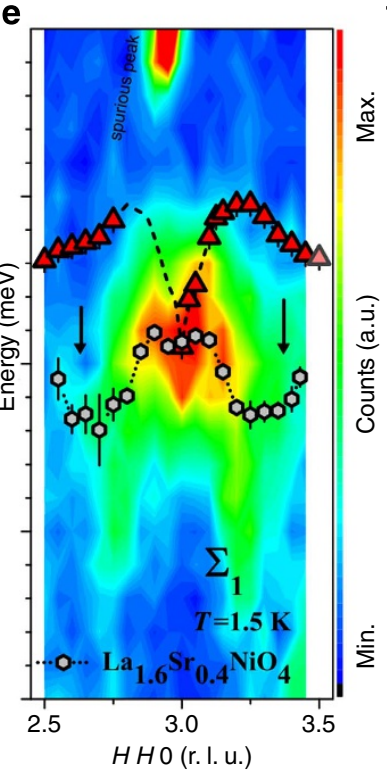

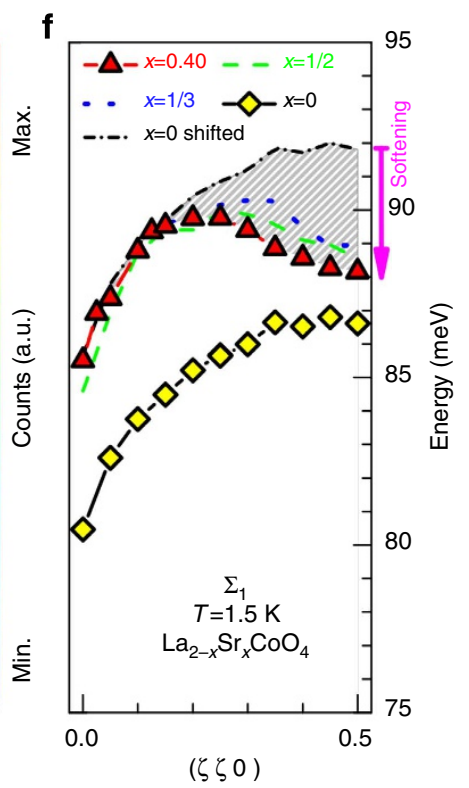

Figure 3 | Inealstic neutron-scattering study of lattice dynamics (a) Polarization patterns for high-frequency $\Sigma_{1}$ Co-O bond-stretching phonon modes. The dashed lines indicate a coupling to charge stripes (left) or to $\mathrm{CBCO}$ (right). (b,c) Some representative phonon $\mathrm{scans}$ of $\mathrm{La}_{1.6} \mathrm{Sr}_{0.4} \mathrm{CoO}_{4}$ measured at $(3-q 3-q 0)$ and for $\mathrm{La}_{1.6} \mathrm{Sr}_{0.4} \mathrm{NiO}_{4}$ measured at $(3+q 3+q 0)$ corresponding to the focusing sides in both compounds. Clearly, the opposite $|q|$ dependence of phonon peak positions can be observed in nickelates and cobaltates. (d) Inelastic neutron-scattering intensity as a function of energy and momentum transfer for $\mathrm{La}_{1.6} \mathrm{Sr}_{0.4} \mathrm{CoO}_{4}$. The white-shaded area is biased by a spurious peak and also measured at the defocusing side. An anomalous phonon softening is clearly observeable at $\zeta=0.5$ rather than at positions indicative for charge stripes (arrows). (e) The corresponding inelastic neutron signal of a $40 \% \mathrm{Sr}$-doped LSNO reference sample with robust diagonal $\mathrm{CSO}_{\text {; }}$ grey hexagons indicate the fitted phonon dispersion. Unlike in $\mathrm{La}_{1.6} \mathrm{Sr}_{0.4} \mathrm{CoO}_{4}$, a phonon softening is observeable around propagation vectors connected to CSO and not for $\zeta \rightarrow 0.5$ where even an upturn is observeable. (f) Comparison of the high-frequency $\Sigma_{1}$ phonon dispersions of $\mathrm{La}_{2}{ }_{-} \mathrm{Sr}_{x} \mathrm{CoO}_{4}$ for different $x$. The black dash-dotted line indicates the dispersion of $\mathrm{La}_{2} \mathrm{CoO}_{4}$ shifted to higher energies. Intensity error bars are statistical error bars calculated by the square root of intensity. Other error bars are s.d. obtained from Gaussian peak fits.

CBCO half-doped system induces additional strong AFM nnexchange interactions. Therefore, the nnn-interactions become ferromagnetic (FM) in total. Without electron doping, these exchange interactions all had been AFM before. To release frustration, the magnetic moments start twisting/spiralling (a spindensity wave appears unlikely in this strongly localized system). The more electrons are doped within a certain given distance in $a$ direction (in $b$ direction), the more centres of frustration and the more spiralling occurs within this certain distance to release frustration. This machanism would be consistent with the increasing incommensurability away from half doping. Further, it is also able to explain the direction of the incommensurate magnetic satellites: as these spiralling effects appear concomitantly in $x$ and in $y$ direction (in-plane), the diagonal direction of the incommensurate satellites arises from the presence of two independent AFM sublattices in the ideal optimum half-doped compound (see ref. 26), both of which are affected together at the same time by the additional doping of one electron; see Fig. $4 \mathrm{~d}$. As there will be the same total twist of spins in $a$ direction and in $b$ direction after a certain distance, the total modulation that is, the propagation appears to be in $[ \pm 1 \pm 1$ 0] direction. Hence, in this particular cobaltate system with two independent AFM sublattices, frustration is able to explain the direction of the incommensurate magnetic satellites as well as the increasing amount of spiralling with increasing electron doping away from half doping, and, thus, the continuously increasing incommensurability. In contrast to our frustration scenario, a charge stripe scenario will probably have problems in explaining the increasing peak broadening of the incommensurate magnetic peaks with decreasing hole doping from $x=1 / 2$ towards $x=1 / 3$, as the most stable diagonal stripe phase with sharpest magnetic reflections can be expected for $x=1 / 3$ as in the nickelate case. However, exactly the opposite can be observed in these layered cobaltates. It is noteworthy that the amount of disorder in the simulations in ref. 23 was introduced fully artificially. A chiral magnetic state has been 
a

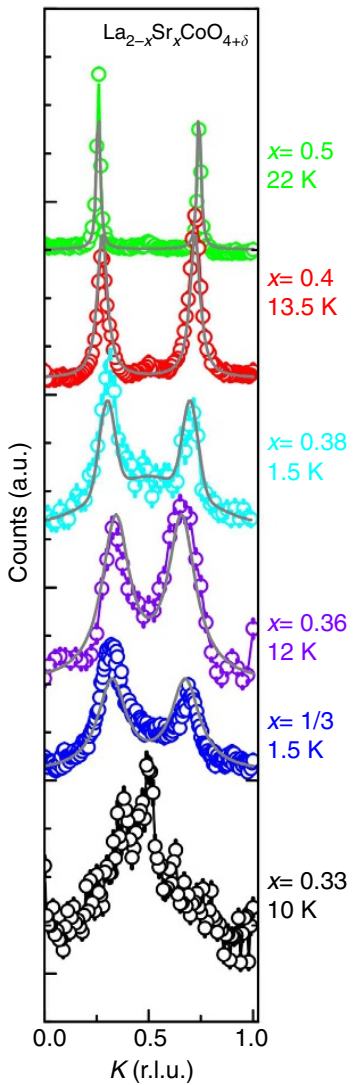

b

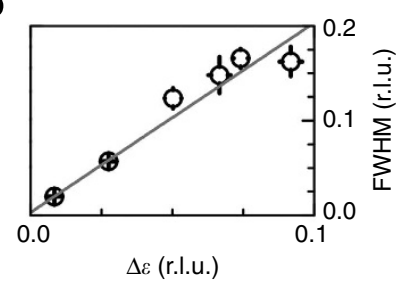

c

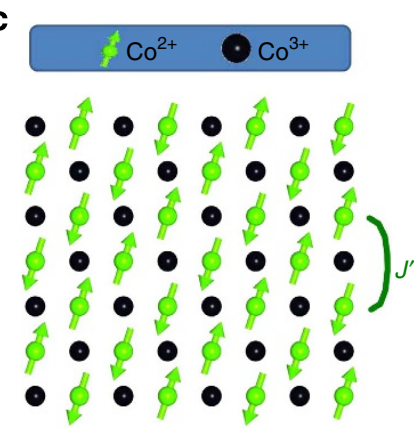

d

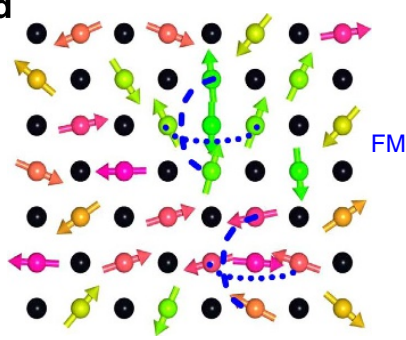

Figure 4 | The frustration scenario in $\mathrm{La}_{2}{ }_{-x} \mathrm{Sr}_{x} \mathrm{CoO}_{4}$. (a) Neutronscattering intensities for $(H \pm H O)$ scans of $\mathrm{La}_{2}{ }_{x} \mathrm{Sr}_{x} \mathrm{CoO}_{4}$ for different $x$ showing a continuous peak broadening below half doping. (b) The peak width for different $x$ as a function of incommensurability $\Delta \varepsilon=\varepsilon-1 / 4$. (c) The AFM structure in an ideal CBCO state. (d) Schematic drawing of our frustration scenario. The introduction of an additional electron $\left(\mathrm{Co}^{2+}\right.$ ion) into the AFM matrix of the ideal $\mathrm{CBCO}$ half-doped compound induces strong frustration because of a strong nn-exchange coupling $J$ compared with the existing exchange couplings $J$ ' within the undistorted AFM matrix. Thus, effectively, one additional FM exchange interaction is introduced into each AFM sublattice of the ideal CBCO spin structure (blue dotted/dashed line). To release frustration, the formation of a chiral or non-collinear magnetic structure appears likely (the change of the spin directions is emphasized by the colour change). Intensity error bars are statistical error bars calculated by the square root of intensity. Other error bars are s.d. obtained from Lorentzian peak fits.

also proposed for the insulating spin glass phase of $\mathrm{La}_{2}{ }_{x} \mathrm{Sr}_{x} \mathrm{CuO}_{4}$ refs 32-34 exhibiting also diagonal magnetic satellites and magnetic excitations with the characteristics of an hour-glass spectrum ${ }^{10}$. In this chiral model of the magnetic ground state of $\mathrm{La}_{2-}{ }_{x} \mathrm{Sr}_{x} \mathrm{CuO}_{4}$, frustration arises from doping of charges into the AFM matrix of an AFM parent compound $\left(\mathrm{La}_{2} \mathrm{CuO}_{4}\right)$ (refs 32-34). Note that also in bulk $\mathrm{La}_{2}-{ }_{x} \mathrm{Sr}_{x} \mathrm{CuO}_{4}$, no CSO peaks have been found ${ }^{35}$, and, that in both isostructural systems ( $M=\mathrm{Cu}$ or Co respectively) frustration might arise from the doping of additional charges (holes or electrons, respectively) to interstitial sites, thus effectively introducing additional FM exchange interactions into the unaltered AFM host structure (see ref. 34 or Fig. 4d, respectively). Hence, frustration might not only have an important role for the triangular lattice ${ }^{36}$ but also for the hole-doped square lattice systems as well as for the emergence of the hour-glass dispersion in these systems.

In conclusion, we studied the magnetic excitations and electron-phonon coupling of a single-layer perovskite cobaltate within the $\mathrm{CBCO}$ regime. In this material with comparably sharp incommensurate magnetic peaks, we observe the appearance of a magnetic excitation spectrum with all basic features of an hourglass spectrum in close vicinity to half doping. The absence of any charge stripe superstructure reflections in this material indicates that the hour-glass dispersion might emerge concomitantly with the onset of frustration and chiral or non-collinear magnetic ordering in $\mathrm{La}_{2}-{ }_{x} \mathrm{Sr}_{x} \mathrm{CoO}_{4}(x \sim 1 / 2)$ rather than with the onset of CSO. We also studied the impact of electron-phonon coupling in these materials and were able to observe large Co-O bondstretching phonon anomalies at the zone boundary, which are consistent with our picture that robust checkerboard charge ordering is still prevalent below half doping in $\mathrm{La}_{2}{ }_{x} \mathrm{Sr}_{x} \mathrm{CoO}_{4}$. Neither the existence of static nor of dynamic charge stripe phases can be supported by these high-frequency phonon studies. Our observation of an hour-glass-shaped excitation spectrum in a stripeless insulating transition metal oxide goes one step further than the recent observation of an hour-glass spectrum in an insulating cobalt oxide ${ }^{8}$, as it clearly shows that besides Fermisurface effects no charge stripes are needed for the appearance of the hour-glass spectrum as well. Therefore, frustration might be the unifying property of all these systems that exhibit an hourglass-shaped magnetic excitation spectrum and either band structure effects or (short ranged) charge stripe correlations are just additional side effects, which are not directly connected to the appearance of this peculiar excitation spectrum.

\section{Methods}

Synthesis. Single crystals of $\mathrm{La}_{2}{ }_{x} \mathrm{Sr}_{x} \mathrm{CoO}_{4}$ and LSNO materials have been grown by the floating zone technique following a route as described elsewhere ${ }^{37,38}$. However, to determin the oxygen content more carefully, we have grown all the crystals in Ar atmosphere. Only one of our single crystals, that is, the nominally $1 / 3$ Sr-doped compound, has been grown in an oxygen-enriched $\mathrm{Ar}$ atmosphere to obtain one single crystal that was grown under more oxidizing conditions like those described for $\mathrm{La}_{5 / 3} \mathrm{Sr}_{1 / 3} \mathrm{CoO}_{4}$ in ref. 8. The high single-crystal quality has been ascertained as described in the Supplementary Note 1.

Neutron scattering. For most inelastic neutron measurements, two or three large single crystals have been co-aligned with the crystallographic $a_{\mathrm{tet}}$ and $b_{\text {tet }}$-axes in the scattering plane. For measuring the magnetic excitation spectra, inelastic neutron-scattering experiments have been performed at the 2T.1 TASs at the LLB in Saclay, France. A vertically focussing configuration of the PG-002 analyser and a flat configuration of the PG-002 monochromator has been chosen and higherorder contributions have been suppressed by two PG filters. All inelastic scans have been performed in the constant $k_{\mathrm{f}}$-operation mode with $k_{\mathrm{f}}=2.662 \AA^{-1}$ in most cases and $k_{\mathrm{f}}=4.1 \AA^{-1}$ for the highest measured energies solely. For measuring the high-frequency $\Sigma_{1}$ phonon dispersion and the constant-energy maps, inelastic neutron-scattering experiments have been performed at the IN8 thermal TAS at the ILL in Grenoble, France. A Cu200 double-focusing monochromator and a PG-002 double-focusing analyser have been chosen for measuring these highenergy phonon modes in constant $k_{\mathrm{f}}$-operation mode with $k_{\mathrm{f}}=2.662 \AA^{-1}$. Higherorder contamination was suppressed using two PG filters. For measuring the constant-energy maps in Fig. 2a, Si-111 monochromator has been used to suppress $\lambda / 2$ contaminations. Powder lines originating from the cryostat and the sample holder are apparent in these measurements. To apply a background correction, we have also performed measurements with empty sample holder (measurements without sample) for the most important constant-energy maps: first, at the neck of the hour-glass, that is, at $20 \mathrm{meV}$, and, second, at a distinctly higher energy of $27 \mathrm{meV}$. Thus, we were able to subtract the background from our sample signal to confirm the shape of our observed magnetic signal. These two maps are shown in Fig. 2n,o. Note that the spurious peaks could still not be corrected this way as they might originate from monochromator phonons that were scattered elastically by the sample etc. The magnon-merging point as well as the outwards dispersion can be clearly seen in these kind of (HK0) maps at 20 and $27 \mathrm{meV}$, respectively. Additional inelastic neutron-scattering experiments of the low-energy magnetic excitations have been performed at the cold neutron TAS 4F.2 at the LLB $\left(k_{\mathrm{f}} \sim 1.55 \AA^{-1}\right.$; Be-filter at $\left.77 \mathrm{~K}\right)$. Elastic neutron-scattering measurements have been performed at the 3T.1 diffractometer and 1T.1 TAS at the LLB and at the IN8 TAS spectrometer at the ILL in Grenoble, France $\left(k_{i} \sim 2.662 \AA^{-1}\right.$; two PG filters have been used for the suppression of higher-order contamination). 


\section{References}

1. Zaanen, J. \& Gunnarsson, O. Charged magnetic domain lines and the magnetism of high- $\mathrm{T}_{\mathrm{c}}$ oxides. Phys. Rev. B 40, 7391-7394 (1989).

2. Schulz, H. J. Domain walls in a doped antiferromagnet. J. Phys. 50, 2833-2849 (1989).

3. Machida, K. Magnetism in $\mathrm{La}_{2} \mathrm{CuO}_{4}$ based compounds. Physica C 158, 192-196 (1989).

4. Tranquada, J. M., Sternlieb, B. J., Axe, J. D., Nakamura, Y. \& Uchida, S. Evidence for stripe correlations of spins and holes in copper oxide superconductors. Nature 375, 561-563 (1995).

5. Tranquada, J. M., Buttrey, D. J., Sachan, V. \& Lorenzo, J. E. Phys. Rev. Lett. 73, 1003-1006 (1994)

6. Tranquada, J. M. et al. Simultaneous ordering of holes and spins in $\mathrm{La}_{2} \mathrm{NiO}_{4.125}$. Phys.Rev.Lett. 78, 338-341 (1997).

7. Cwik, M. et al. Magnetic correlations in $\mathrm{La}_{2}{ }_{\mathrm{x}} \mathrm{Sr}_{\mathrm{x}} \mathrm{CoO}_{4}$ studied by neutron scattering: possible evidence for stripe phases. Phys. Rev. Lett. 102, 057201 (2009).

8. Boothroyd, A. T., Babkevich, P., Prabhakaran, D. \& Freeman, P. G. An hourglass magnetic spectrum in an insulating, hole-doped antiferromagnet. Nature 471, 341-344 (2011).

9. Tranquada et al. Quantum magnetic excitations from stripes in copper oxide superconductors. Nature 429, 534-538 (2004).

10. Matsuda, M. et al. Magnetic dispersion of the diagonal incommensurate phase in lightly doped $\mathrm{La}_{2}{ }_{\mathrm{x}} \mathrm{Sr}_{\mathrm{x}} \mathrm{CuO}_{4}$. Phys. Rev. Lett. 101, 197001 (2008).

11. Arai, M. et al. Incommensurate spin dynamics of underdoped superconductor $\mathrm{YBa}_{2} \mathrm{Cu}_{3} \mathrm{O}_{6.7}$. Phys. Rev. Lett. 83, 608-611 (1999).

12. Bourges, P. et al. The spin excitation spectrum in superconducting $\mathrm{YBa}_{2} \mathrm{Cu}_{3} \mathrm{O}_{6.85}$. Science 288, 1234-1237 (2000).

13. Christensen, N. B. et al. Dispersive excitations in the high-temperature superconductor $\mathrm{La}_{2}{ }_{x} \mathrm{Sr}_{\mathrm{x}} \mathrm{CuO}_{4}$. Phys. Rev. Lett. 93, 147002 (2004).

14. Hayden, S. M., Mook, H. A., Dai, P., Perring, T. G. \& Dogan, F. The structure of the high-energy spin excitations in a high-transition-temperature superconductor. Nature 429, 531-534 (2004).

15. Vignolle, B. et al. Two energy scales in the spin excitations of the hightemperature superconductor $\mathrm{La}_{2}-\mathrm{Sr}_{\mathrm{x}} \mathrm{CuO}_{4}$. Nat. Phys. 3, 163-167 (2007)

16. Xu, G. et al. Testing the itinerancy of spin dynamics in superconducting $\mathrm{Bi}_{2} \mathrm{Sr}_{2} \mathrm{CaCu}_{2} \mathrm{O}_{8+\delta}$. Nat. Phys. 5, 642-646 (2009).

17. Lipscombe, O. J., Vignolle, B., Perring, T. G., Frost, C. D. \& Hayden, S. M. Emergence of coherent magnetic excitations in the high temperature underdoped $\mathrm{La}_{2}-\mathrm{Sr}_{\mathrm{x}} \mathrm{CuO}_{4}$ superconductor at low temperatures. Phys. Rev. Lett. 102, 167002 (2009).

18. Hinkov, V. et al. Spin dynamics in the pseudogap state of a high-temperature superconductor. Nat. Phys. 3, 780-785 (2007).

19. Eremin, I., Morr, D. K., Chubukov, A. V., Bennemann, K. H. \& Norman, M. R. Novel neutron resonance mode in $\mathrm{d}_{\mathrm{x} 2-\mathrm{y} 2}$-wave superconductors. Phys. Rev. Lett. 94, 147001 (2005).

20. Vojta, M., Vojta, T. \& Kaul, R. K. Spin excitations in fluctuating stripe phases of doped cuprate superconductors. Phys. Rev. Lett. 97, 097001 (2006).

21. Seibold, G. \& Lorenzana, J. Magnetic fluctuations of stripes in the high temperature cuprate superconductors. Phys. Rev. Lett. 94, 107006 (2005).

22. Andersen, B. M., Graser, S. \& Hirschfeld, P. J. Disorder-induced freezing of dynamical spin fluctuations in underdoped cuprate superconductors. Phys. Rev. Lett. 105, 147002 (2010).

23. Andrade, E. C. \& Vojta, M. Disorder, cluster spin glass, and hourglass spectra in striped magnetic insulators. Phys. Rev. Lett. 109, 147201 (2012).

24. Sakiyama, N., Zaliznyak, I. A., Lee, S.-H., Mitsui, Y. \& Yoshizawa, H. Dopingdependent charge and spin superstructures in layered cobalt perovskites. Phys. Rev. B 78, 180406(R) (2008).
25. Rotter, M., Le, D. M., Boothroyd, A. T. \& Blanco, J. A. Dynamical matrix diagonalization for the calculation of dispersive excitations. J. Phys. Cond. Mat 24, 213201 (2012).

26. Helme, L. M. et al. Magnetic order and dynamics of the charge-ordered antiferromagnet $\mathrm{La}_{1.5} \mathrm{Sr}_{0.5} \mathrm{CoO}_{4}$. Phys. Rev. B 80, 134414 (2009).

27. Matsuda, M., Granroth, G. E., Fujita, M., Yamada, K. \& Tranquada, J. M. Energy-dependent crossover from anisotropic to isotropic magnetic dispersion in lightly doped $\mathrm{La}_{1.96} \mathrm{Sr}_{0.0} 4 \mathrm{CuO}_{4}$. Phys. Rev. B 87, 054508 (2013).

28. Rømer, A. T. et al. Glassy low-energy spin fluctuations and anisotropy gap in $\mathrm{La}_{1.88} \mathrm{Sr}_{0.12} \mathrm{CuO}_{4}$. Phys. Rev. B 87, 144513 (2013).

29. Reznik, D. et al. Electron-phonon coupling reflecting dynamic charge inhomogeneity in copper oxide superconductors. Nature 440, 1170 (2006).

30. Komarek, A. C. Complex ordering phenomena in transition metal oxides and oxyhalides. PhD thesis, Universität zu Köln (2009).

31. Zaanen, J. High-temperature superconductivity: the secret of the hourglass. Nature 471, 314-316 (2011).

32. Sushkov, O. P. \& Kotov, V. N. Theory of incommensurate magnetic correlations across the insulator-superconductor transition of underdoped $\mathrm{La}_{2}{ }_{\mathrm{x}} \mathrm{Sr}_{\mathrm{x}} \mathrm{CuO}_{4}$. Phys. Rev. Lett. 94, 097005 (2005).

33. Lüscher, A., Milstein, A. I. \& Sushkov, O. P. Structure of the spin-glass state of $\mathrm{La}_{2}{ }_{\mathrm{x}} \mathrm{Sr}_{\mathrm{x}} \mathrm{CuO}_{4}$ : the spiral theory. Phys. Rev. Lett. 98, 037001 (2007).

34. Hasselmann, N., Castro Neto, A. H. \& Morais Smith, C. Spin-glass phase of cuprates. Phys. Rev. B 69, 014424 (2004).

35. Wu, H.-H. et al. Charge stripe order near the surface of 12-percent doped $\mathrm{La}_{2}{ }_{\mathrm{x}} \mathrm{Sr}_{\mathrm{x}} \mathrm{CuO}_{4}$. Nat. Commun 3, 1023 (2012).

36. Takada, K. et al. Superconductivity in two-dimensional $\mathrm{CoO}_{2}$ layers. Nature 422, 53-55 (2003).

37. Matsuura, T., Mizusaki, J., Yamauchi, S. \& Fueki, K. Single crystal growth of $\mathrm{La}_{2}{ }_{\mathrm{x}} \mathrm{Sr}_{\mathrm{x}} \mathrm{CoO}_{4}(\mathrm{x}=0.0,0.5,1.0$ and 1.5). Jpn J. Appl. Phys. 23, 1143-1144 (1984).

38. Jang, W.-J. \& Takei, H. Growth, structure and properties of $\mathrm{La}_{2}{ }_{\mathrm{x}} \mathrm{Sr}_{\mathrm{x}} \mathrm{NiO}_{4}$ $(\mathrm{x}=0-0.3)$ single crystals. Jpn J. Appl. Phys. 30, 251-257 (1991).

\section{Acknowledgements}

We thank Y. Sidis, D.I. Khomskii, E. Andrade, M. Rotter, P. Thalmeier, O. Stockert and L.H. Tjeng for their helpful discussions. We thank H. Borrmann, Y. Prots and S.Hückmann for X-ray diffraction measurements. We also thank S. Subakti for titration measurements and A. Keil for help in cutting crystals. We thank the team of U. Burkhardt for EDX measurements and the team of G. Auffermann for ICP measurements.

\section{Author Contributions}

A.C.K. planned all experiments, synthesized all studied materials and wrote the manuscript Y.D. and D.L. performed the elastic and inelastic neutron-scattering experiments at the 3T.1 diffractometer and at the $2 \mathrm{~T}$ and $1 \mathrm{~T}$ spectrometers. Y.D., A.P. and A.C.K. performed the elastic and inelastic neutron-scattering experiments at the IN8 spectrometer.

\section{Additional Information}

Supplementary Information accompanies this paper on http://www.nature.com/ naturecommunications

Competing financial interests: The authors declare no competing financial interests.

Reprints and permission information is available online at http://npg.nature.com/ reprintsandpermissions/

How to cite this article: Drees, Y. et al. Hour-glass magnetic spectrum in a stripeless insulating transition metal oxide. Nat. Commun. 4:2449 doi: 10.1038/ncomms3449 (2013). 